ARTICLE

https://doi.org/10.1038/s41467-019-12449-2

\title{
High levels of AAV vector integration into CRISPR- induced DNA breaks
}

Killian S. Hanlon 1,2, Benjamin P. Kleinstiver (10 3,4,5, Sara P. Garcia (D) 5,6,7, Mikołaj P. Zaborowski (D) 2,8,9, Adrienn Volak ${ }^{2,10}$, Stefan E. Spirig ${ }^{10}$, Alissa Muller ${ }^{10}$, Alexander A. Sousa6,7, Shengdar Q. Tsai (1) ${ }^{11}$, Niclas E. Bengtsson ${ }^{12}$, Camilla Lööv ${ }^{13}$, Martin Ingelsson ${ }^{13}$, Jeffrey S. Chamberlain ${ }^{12}$, David P. Corey ${ }^{1}$, Martin J. Aryee (1) $5,6,7,14$, J. Keith Joung (1) 5,6,7, Xandra O. Breakefield ${ }^{2,8}$, Casey A. Maguire ${ }^{2,8 \star}$ \& Bence György (10) 1,2,10*

Adeno-associated virus (AAV) vectors have shown promising results in preclinical models, but the genomic consequences of transduction with AAV vectors encoding CRISPR-Cas nucleases is still being examined. In this study, we observe high levels of AAV integration (up to $47 \%$ ) into Cas9-induced double-strand breaks (DSBs) in therapeutically relevant genes in cultured murine neurons, mouse brain, muscle and cochlea. Genome-wide AAV mapping in mouse brain shows no overall increase of AAV integration except at the CRISPR/Cas9 target site. To allow detailed characterization of integration events we engineer a miniature AAV encoding a $465 \mathrm{bp}$ lambda bacteriophage DNA (AAV- $\lambda 465$ ), enabling sequencing of the entire integrated vector genome. The integration profile of $A A V-465 \lambda$ in cultured cells display both full-length and fragmented AAV genomes at Cas9 on-target sites. Our data indicate that AAV integration should be recognized as a common outcome for applications that utilize AAV for genome editing.

\footnotetext{
${ }^{1}$ Department of Neurobiology, Harvard Medical School, Boston, MA 02115, USA. ${ }^{2}$ Molecular Neurogenetics Unit, Department of Neurology, Massachusetts General Hospital, Charlestown, MA 02129, USA. ${ }^{3}$ Center for Genomic Medicine, Massachusetts General Hospital, Boston, MA, USA. ${ }^{4}$ Department of Pathology, Massachusetts General Hospital, Boston, MA, USA. ${ }^{5}$ Department of Pathology, Harvard Medical School, Boston, MA, USA. ${ }^{6}$ Molecular Pathology Unit, Massachusetts General Hospital, Charlestown, MA 02129, USA. ${ }^{7}$ Center for Cancer Research and Center for Computational and Integrative Biology, Massachusetts General Hospital, Charlestown, MA, USA. ${ }^{8}$ Program in Neuroscience, Harvard Medical School, Boston, MA 02115, USA. ${ }^{9}$ Department of Gynecology, Obstetrics and Gynecologic Oncology, Division of Gynecologic Oncology, Poznań University of Medical Sciences, 60-535 Poznań, Poland. 10 Institute of Molecular and Clinical Ophthalmology Basel, 4031 Basel, Switzerland. ${ }^{11}$ Department of Hematology, St. Jude Children's Research Hospital, Memphis, TN, USA. ${ }^{12}$ Department of Neurology, University of Washington, Seattle, WA 98195, USA. ${ }^{13}$ Uppsala University, Department of Public Health and Caring Sciences, Geriatrics, Uppsala, Sweden. ${ }^{14}$ Department of Biostatistics, Harvard T. H. Chan School of Public Health, Boston, MA, USA.

*email: cmaguire@mgh.harvard.edu; bence.gyoergy@iob.ch
} 
G enome editing with RNA-guided nucleases holds great promise for the treatment of human diseases. Recently, there have been several advances in addressing some of the key challenges in the case of in vivo genome editing, such as increasing specificity ${ }^{1-3}$ and developing delivery tools for in vivo applications $s^{4,5}$. In particular, delivery of CRISPR/Cas9 nucleases by AAV vectors has shown therapeutic benefit in multiple preclinical models of diseases ${ }^{6-10}$ and this modality is moving quickly towards clinical trials ${ }^{11}$. AAV offers safe and stable transgene expression levels in vivo in many differentiated tissues, and the recent FDA approval of the AAV-based gene therapy product Luxturna places this vector at the forefront of gene therapy. However, long-term expression of certain transgenes (e.g., Cas9) from an AAV vector might lead to genotoxic effects due to the sustained activity of an active nuclease. Currently, there is limited in-depth characterization of potential outcomes of AAV-mediated CRISPR delivery, particularly in target tissues after in vivo administration. It is well known that the majority of AAV vectors exist in an extrachromosomal state, however, it has been shown previously that a fraction of AAV vectors integrate into pre-existing double-stranded breaks (DSB) ${ }^{12,13}$. Furthermore, non-homologous end-joining (NHEJ)-mediated integration of AAV vectors was also observed after DSBs induced by zinc-finger nuclease or CRISPR in liver ${ }^{14}$, and in muscle ${ }^{15}$, and eye $^{11}$, respectively. There has been limited in-depth characterization of AAV integration into nuclease-induced breaks in nondividing cells in vivo.

In this study, we analyze integration of AAV vectors genomewide and into CRISPR-induced DSBs in vivo focusing on therapeutically relevant target genes in differentiated cells of the nervous system $(A P P S W, M e c p 2, D n m t 3 b)$, muscle $(D m d)$ and the inner ear (Tmc1). APP and human TMC1 are carriers of important dominantly inherited mutations that cause early-onset Alzheimer's disease and progressive deafness ${ }^{16}$, respectively, whereas MECP2 is a potential target gene in Rett syndrome ${ }^{17}$. DNMT3B is expressed during neurodevelopment and is involved in DNA methylation ${ }^{18}$. Gene editing is a very promising strategy for Duchenne muscular dystrophy, as exon deletion or homologous recombination after CRISPR targeting can restore dystrophin expression with improvement in muscle function $6,15,19-21$. Surprisingly, we observe high integration frequencies of AAV sequences at the CRISPR cut sites of all on-target genes. Using miniature AAV vectors we demonstrate that integrated vector genomes can be fragmented, full length, or be present as concatemers. In vivo genome-wide mapping of AAV vectors in the brain shows integration into multiple sites, including the CRISPR on-target site. However, outside of the CRISPR target region, genome-wide AAV integration rates are not different between an AAV control vector and AAV carrying Cas9 and gRNA, suggesting that Cas9 does not lead to widespread genotoxic effects in the brain.

\section{Results}

AAV vector sequences are detected at CRISPR-induced DSBs. In analyzing next-generation sequencing (NGS) data from an in vivo CRISPR gene therapy approach targeting the $T m c 1^{\text {Bee- }}$ thoven mutation in the inner ear, we observed AAV inverted terminal repeat (ITR) sequences within CRISPR indels ${ }^{22}$. To confirm this observation and expand these findings to other genes, we first analyzed AAV vector integration in cultured cortical neurons derived from wild type (WT) C57BL/6 mice. Cells were treated with AAV1 carrying S. pyogenes Cas9 (SpCas9) and separate AAV1 vectors carrying gRNAs against the wild type (WT) coding sequence of Mecp2, Dnmt3b or Tmc1, at $10^{5}$ or $10^{6}$ vector genomes $(\mathrm{vg})$ per cell. Cells were incubated with AAV vectors for 1 day and then kept in culture for another 20 days before harvesting genomic DNA to assess AAV integration (Fig. 1a). Genomic DNA was amplified by a high-fidelity polymerase using a very long extension time (see Methods) to allow for inclusion of potentially large integration events. Deep sequencing of PCR products from the region flanking the cut site revealed characteristic indel formation in all targeted genes (Fig. 1a and Supplementary Data 1), with the majority of indels observed being single nucleotide changes. Analysis of the percentage of reads that aligned to AAV sequences revealed that for all target genes, fusion reads between AAV vector genome and host genome were detectable at variable efficiencies $(0.06-12.5 \%$; Fig. 1a). To quantify AAV integration efficiency as a fraction of the total nuclease-induced events, reads that contained AAV sequences were normalized against all reads that harbored insertions or deletions compared with the reference sequence (including AAV integration). This measure is termed AAV capture ratio. We found the AAV capture ratio varied between $13.8 \%$ and $36.5 \%$ among different targets (Fig. 1a) and was not significantly different between the two different $\mathrm{vg} / \mathrm{cell}$ conditions (paired $t$-test, $p=0.224$ ). As expected, the higher dose of AAVCas9 and AAV-gRNA led to a higher percentage of reads with indels. For example at $10^{5} \mathrm{gc} / \mathrm{cell}$ with the Tmcl specific gRNA, there were $18.0 \%$ of reads with indels, while this increased to $49.8 \%$ at $10^{6} \mathrm{gc} / \mathrm{cell}$. We also reanalyzed genomic DNA from our previous study 8 using mouse primary cortical neurons, which overexpress a mutated form of human APP gene (amyloid precursor protein with the Swedish mutation, $A P P^{S W}$ from the Tg2576 strain). In this study ${ }^{8}$, the experimental conditions were the same as in the present study. Similarly, we observed AAV integration into $A P P^{S W}$, with capture ratios of $20.6 \%$ and $27.1 \%$ for $10^{5}$ or $10^{6} \mathrm{vg} / \mathrm{cell}$, respectively. These results suggest that a substantial number of gene editing outcomes in non-dividing cultured neurons are a result of AAV vector integration at the ontarget site.

Vector integration into therapeutically relevant genes in vivo. Next, we analyzed AAV vector integration into CRISPR-induced breaks in vivo in three different organs (brain, cochlea, and muscle). We first analyzed in vivo AAV integration in the brain by performing intrahippocampal injections of separate AAV1 vectors expressing Cas 9 and gRNAs targeting either the Mecp2 or Dnmt3b genes $\left(5 \times 10^{9} \mathrm{vg}\right.$ from AAV-Cas 9 and $3 \times 10^{9} \mathrm{vg}$ from AAV-gRNA). Similar to above, we also reanalyzed genomic DNA isolated from hippocampus tissue from human $A P P S W$ transgenic mice (Tg2576) treated with AAVs encoding Cas9 and a gRNA targeting the $A P P S W$ gene ${ }^{8}$. We observed indel formation and $\mathrm{AAV}$ integration at the CRISPR on-target site for all three target genes in vivo in the hippocampus (Fig. 1b and Supplementary Data 2). AAV capture ratios (reads with AAV integration normalized to reads with indels) were found to be $39.3 \%, 10.8 \%$, and $32.3 \%$ for $M e c p 2, D n m t 3 b$, and $A P P^{S W}$, respectively.

In the cochlea, we analyzed data from our previous study ${ }^{22}$ and found AAV integration into Tmc1 (Fig. 1b). In this study, an allele-selective S. aureus $\mathrm{Cas} 9\left(\mathrm{SaCas} 9-\mathrm{KKH}^{23}\right)$ was used to target the Beethoven ${ }^{16}$ mutation, and AAV-mediated allele-selective disruption led to a complete halt of hair cell degeneration and hearing preservation up to 1 year post injection ${ }^{22}$. Integration was found in the $T m c 1$ gene in injected animals with a capture ratio of $26.6 \%$ (Fig. 1b).

We also analyzed genomic DNA from the study by Bengtsson et al. ${ }^{6}$ to assess AAV integration in muscle tissue in vivo. In this study, mice received intravenous injections of AAVs carrying either SpCas9 or S. aureus Cas9 (SaCas9) and gRNAs targeting the Dmd gene. We observed AAV integration in all CRISPR target sites, including SaCas9 and SpCas9 target sites 
a

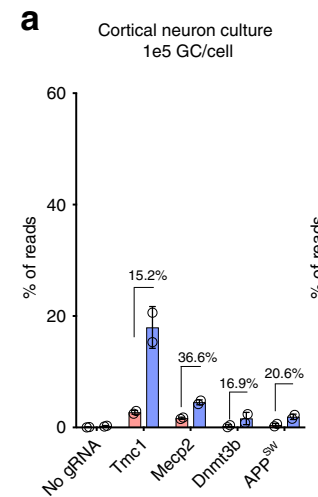

C
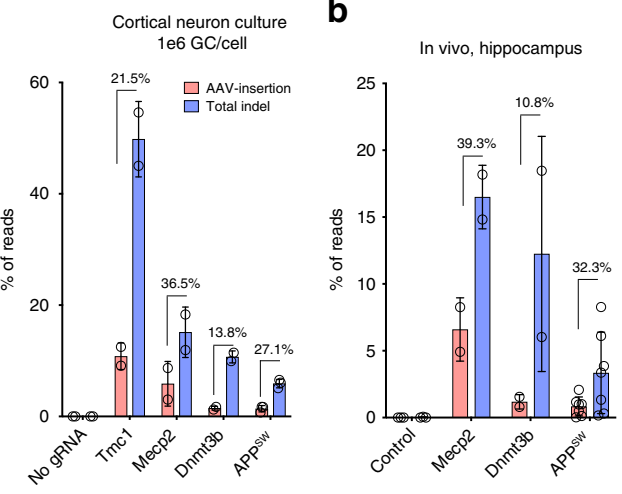

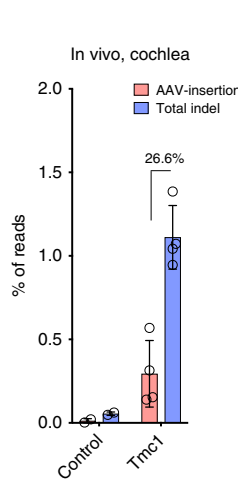

In vivo, muscle In vivo, muscle
(i51-i53 PCR)

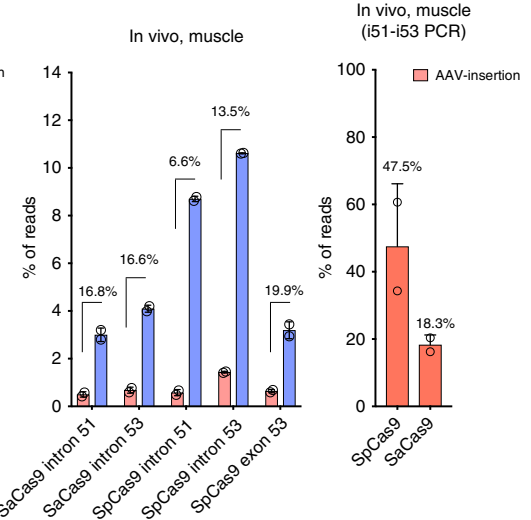

CRISPResso analysis, Dnmt3b, in vivo, hippocampus
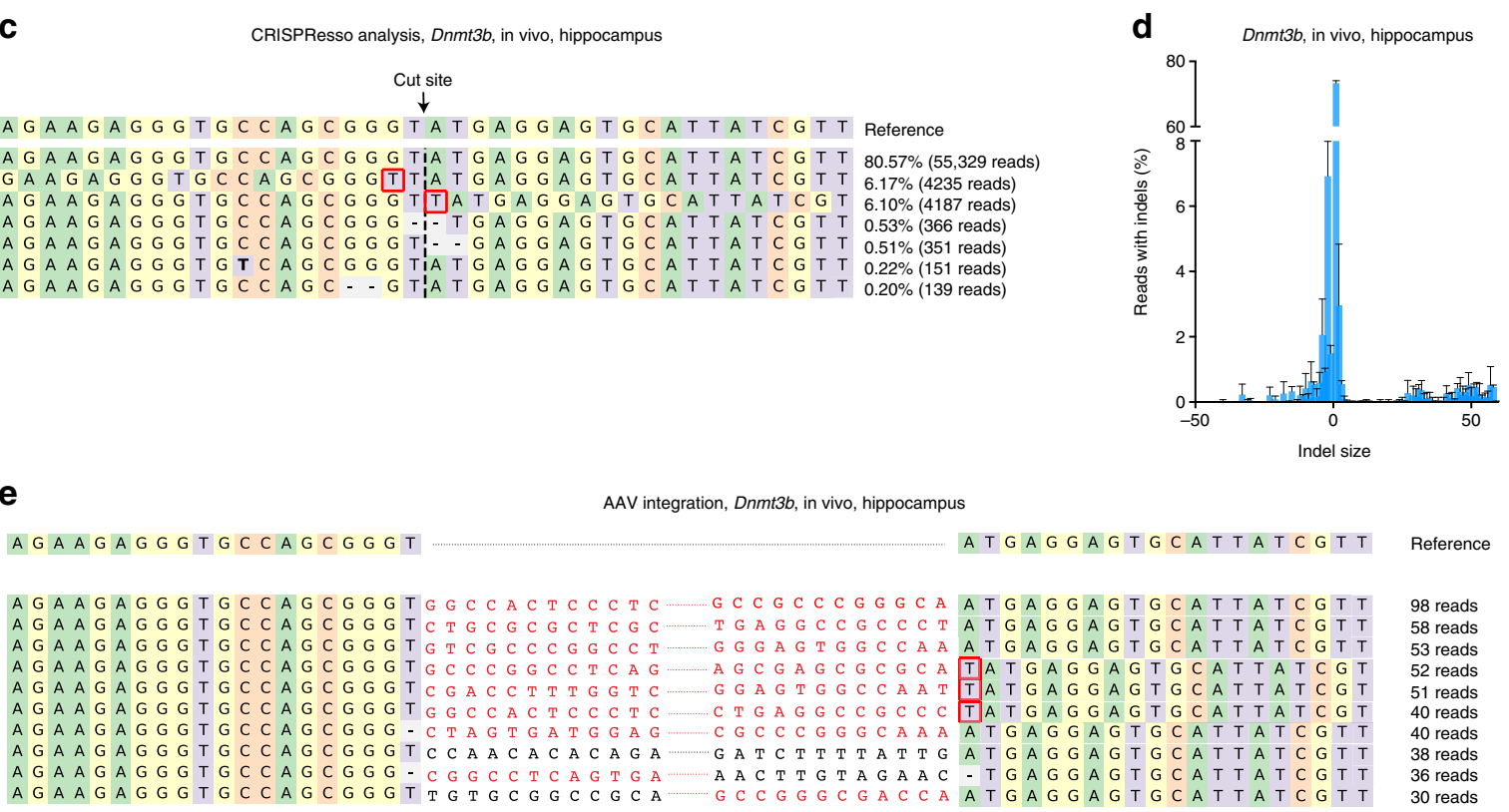

f

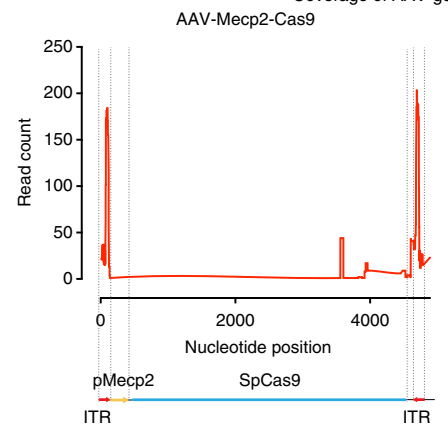

$\underset{\substack{\text { Cut site } \\ \downarrow}}{\downarrow}$

A G A A A G GGTGCCAGCGGGTATGAGGAGTGCATTATCGTT Reference A G A A G A G G G T G C C A G C G G G TiA T G A G G A G T G C A T T A T C G T T $80.57 \%(55,329$ reads) G A A G A G G G T G C C A G C G G GTT T A T G A G G A G T G C A T T A T C G T T $6.17 \%$ (4235 reads) A G A A G A G G G T G C C A G C G G G T T A T G A G G A G T G C A T T A T C G T $6.10 \%$ (4187 reads) A G A A G A G G G T G C C A G C G G G - T- T G A G G A G T G C A T T A T C C G T T T $0.53 \%$ (366 reads) A G A A G A G G G T G C C A G C G G G T - - G A G G A G T G C A T T A T C G T T $0.51 \%$ (351 reads) $\begin{array}{lllllllllll}\text { A G A A G A G G T G T C A G C G G T TA T G A G G A G T G C A T T A T C G T T } & 0.22 \% \text { (151 reads) } \\ \text { A G A A G A G G G T G C C A G C - G T T T G A G G A G T G C A T T A T C G T T } & 0.20 \% \text { (139 reads) }\end{array}$

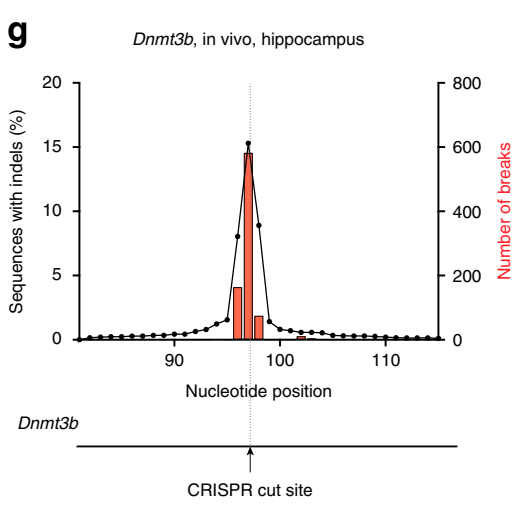

in introns 51 and 53 and in exon 53 (Fig. 1b). Deleting exons 52 and 53 is one of the applied therapeutic strategies by Bengtsson et al. 6 . We wondered whether we could detect AAV sequences when the large $\sim 45 \mathrm{~kb}$ genomic DNA region containing exons 52 and 53 is deleted; thus we performed a PCR with primers in introns 51 and 53. Sequencing this PCR product revealed the anticipated $45 \mathrm{~kb}$ deletion, but also high levels of AAV integration between the cut sites (Fig. 1b, and Supplementary Data 3) with up to $47.5 \%$ efficiency.

Altogether these results confirm that AAV integration is a common occurrence after Cas9-induced breaks in vivo in brain, cochlea and muscle.
Genome editing events after AAV-mediated CRISPR delivery. Next, we set out to more thoroughly characterize the molecular outcomes of genome editing and AAV vector integration in the treated hippocampus samples. Analysis of the size of indels occurring at the Dnmt3b on-target site revealed a bimodal distribution of relatively small deletions and longer insertions (Fig. 1c-e). While the majority of indels were relatively small $(<25 \mathrm{nt})$ in size $(92.3 \pm 1.4 \%)$, we also observed insertions longer than $25 \mathrm{nt}(7.3 \pm 1.6 \%$ of all indels; Fig. 1d, e). By aligning the long insertion reads to the AAV genome, we identified that these larger insertion events were AAV vector integrations (Fig. 1e). The vast majority (97.8\%) of the junction sites between Dnmt3b 
Fig. $1 \mathrm{AAV}$ vectors integrate into CRISPR/Cas9 cut sites in vitro and in vivo. a Primary murine cortical neurons were transduced with an AAV1 vector encoding Cas9 as well as another AAV1 vector encoding a guide RNA (gRNA) against the genes indicated. Two different doses, 1e5 gc/cell (left panel) and 1 e6 gc/cell (right panel), were tested. The negative control was neurons transduced by AAV-Cas9 vector without gRNA (Tmc1 gene was amplified by PCR for $1 \mathrm{e} 5 \mathrm{gc} / \mathrm{cell}$ and APP gene was amplified for $1 \mathrm{e} 6 \mathrm{gc} / \mathrm{cell})$. Frequency of AAV sequences present at indels at the target site are shown in red vs total number of indels in blue. AAV capture efficiencies are shown as percentages on the graphs. Two biological replicates were sequenced for each condition (3 for APPSW gene, 1 e6 gc/cell dose). b AAV integration into Cas9 cut sites targeting therapeutic genes in the murine hippocampus, cochlea or muscle. For $A P P S W$, non-injected cerebellum or cortex was used as control. For Tmc1, non-injected cochleas were used. Animal numbers and the number of sequencing reactions are as follows (numbers of animals pooled per reaction included in parentheses): Hippocampus, control: $n=3$ ( 3 reactions), Mecp2: $n=5$ ( 2 reactions, $n=3$ and 2), Dnmt3b: $n=5$ (2 reactions, $n=3$ and 2), APPSW: $n=7$ ( 7 reactions). Cochlea samples, non-injected: $n=21$ ( 2 reactions, $n=9$ and 12), injected: $n=33$ ( 4 reactions, $n=6,6,9$, and 12 animals). Muscle samples: $n=8$ (2 reactions, $n=4$ and 4). c CRISPResso analysis showing small indels at cut site from hippocampus, injected with AAV-Cas9 and AAV-gRNA against Dnmt3b. d Bimodal distribution of indel sizes, the larger indicating $\mathrm{AAV}$ sequence integration at the cut site, with specific examples shown in e (two sequencing reactions from 2 and 3 animals, respectively).

f Characterization of AAV vector region present in indels with AAV-Mecp2-Cas9 (left panel) and AAV-U6-gRNA-syn-GFP (right panel) in brain samples (Dnmt3b was targeted). $\mathbf{g}$ Distribution of AAV integration surrounding the CRISPR cut site in the case of hippocampus, Dnmt3b was targeted (two sequencing reactions from 2 and 3 animals, respectively). Bars represent mean \pm SD. Source data are provided as a Source Data file

and AAV were within $1 \mathrm{bp}$ of the CRISPR cut site ( 3 bp upstream of the protospacer adjacent motif (PAM) site), suggesting that this AAV integration was indeed at the expected CRISPR-induced break site (Fig. 1e). Additionally, the majority (83.5\%) of AAVgenomic junctions contained elements of the viral ITRs (Fig. 1e, f). However, it was not possible to determine the length and efficiency of AAV vector genome integration events due to the relatively short NGS read length (i.e., longer integrants containing ITR and other components of the AAV cassette may not be detected or detected at lower frequencies (Fig. 1f). By examining the genomic location of small indels and AAV-genomic junction sites, we confirmed the two types of events overlap suggesting that AAV integrates precisely at the cut site, but not around it (Fig. 1g). Furthermore, these results show that the majority of AAV-genomic junctions contain sequences of the ITR elements in vivo.

Genome-wide AAV mapping from mouse brain. Next, we wondered whether the expression of Cas9 and/or gRNA would facilitate AAV integration into genomic sites outside of the target locus. To map AAV integrations within the genome in the brain, we injected mice into the hippocampus with AAV1 vectors encoding for Cas9 and/or gRNA and performed deep sequencing of AAV-genomic junctions. To locate AAV integration sites, we used a modified version of the GUIDE-Seq ${ }^{24}$ pipeline adapted for AAV ITRs with primers specific for the ' $a$ ' region (Supplementary Fig. 1). We injected mice with AAV1 vectors encoding Cas9 and gRNAs targeting Mecp2, Dnmt3b, and $A P P S W$ sequences and 6 weeks after injection, we collected the hippocampus and isolated genomic DNA.

Integration sites were mapped with Virus-Clip ${ }^{25}$ and output was filtered to exclude false positive hits. We excluded sites that showed homology to the vector sequence or sites that only showed genomic sequences, but no viral ITR elements (for full details see Methods section). For a full list of all the sites, including excluded sites and reasons for exclusion, see Supplementary Data files 4-9. For all true integration sites showing genomic alignments see Supplementary Data 10-15 and Supplementary Data 16.

First, we analyzed the global integration profile of AAV in all samples and identified 11-19 unique integration sites per condition. We did not observe an increase in the overall number of integration sites when Cas9 and gRNA were both present (Fig. 2a). The majority of the integration sites were found to be intronic or intergenic, with an average of $44.7 \%$ and $33.4 \%$, respectively. Exonic and regulatory region (promoter, downstream) integrations were rather rare $(3.5 \%$ and $9.2 \%$, respectively). In mice co-injected with AAV-Cas9 and AAV-gRNA vectors, we observed AAV integration in all three CRISPR target sites. Next, we analyzed the total integrant read counts normalized to the total number of sequenced reads (Fig. 2b). Total integration efficiency was the highest in animals treated with AAV-Cas9 and AAV-gRNA against APPSW (Fig. 2b, $82.0 \%$ and $92.4 \%$ of integrants were found in the on-target region). This is not surprising as $A P P^{S W}$ is a transgenic mouse with multiple copies of the human transgene in a mouse genome. In the case of Mecp2 and Dnmt3b targets, 13.4 and $10.7 \%$ of integrants were in the on-target locus. Importantly, AAV integration also occurred in AAV-Cas9 only and AAV-gRNA only conditions with a similar level to the AAV-Cas9 + AAV-gRNA Dnmt3b condition.

Finally, we plotted all integration sites from all conditions and analyzed whether we could identify common sites. Integration sites appeared very variable and not consistent even between the two animals treated with AAV-Cas9 and AAV-gRNA ${ }^{\text {APPSW }}$ (Fig. 2c, d). However, we identified sites that favored AAV integration (Fig. 2c). These included Mgat5b (intronic, detected in 4/6 conditions), Pgm5 (intronic, detected in $4 / 6$ conditions), a region of chr13 (97 MB) (intergenic, detected in 4/6 conditions), Aagab (intronic, detected in $3 / 6$ conditions), a region of chr7, $72 \mathrm{MB}$ (intergenic, detected in $3 / 6$ conditions), Nrip 2 (exonic, detected in $3 / 6$ conditions), and Tex14 (intronic, detected in 3/6 conditions) (Fig. 2c, d). None of the identified sites showed homology to the gRNA target region, suggesting that AAV integration into predicted off-target cut sites was below the level of detection.

Taken together, these results suggest that the presence of Cas9 alone or the presence of Cas9 and gRNA co-delivered by AAV does not influence the genome-wide integration efficiency of AAV (compared with the AAV-gRNA alone vector), except at the CRISPR target site.

\section{A miniaturized AAV allows characterization of CRISPR DSBs.} The profile of AAV integration in CRISPR cut sites determined by NGS appears to favor the ITR region. However, due to the limitations of the read length of NGS (the size of the Cas9 encoding vector is over $4 \mathrm{~kb}$ and the gRNA vector over $2 \mathrm{~kb}$ ), the profile generated in Fig. If is likely to be biased toward the periphery of the AAV vector genome, where ITRs are located. Thus, the question of whether AAV integration is occurring preferentially in the ITR region, or whether the AAV vector integrant is full length or fragmented, could not have been determined. In order to overcome this issue, we designed a minimal AAV construct, in which a very short cargo (175 bp) is flanked by ITR elements (Fig. 3a). For stuffer DNA, we chose a region of the $\lambda$ bacteriophage genome which is highly orthologous to the human/ mouse genome. Together with the ITR elements, we synthesized and cloned this short vector, termed AAV- $\lambda 465$ (465 bp). 
a

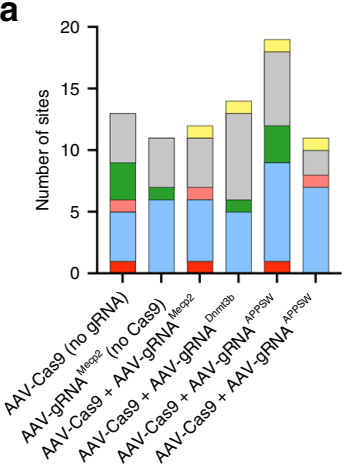

C
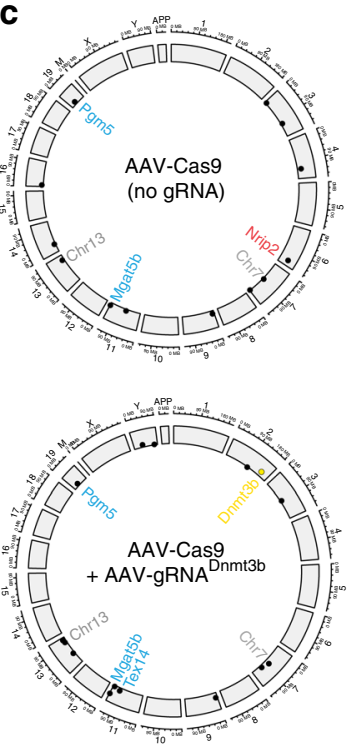

d

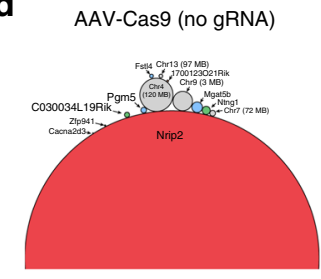

AAV-Cas9 + AAV-gRNA ${ }^{\text {Dnmt3b }}$

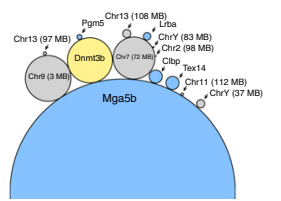

b

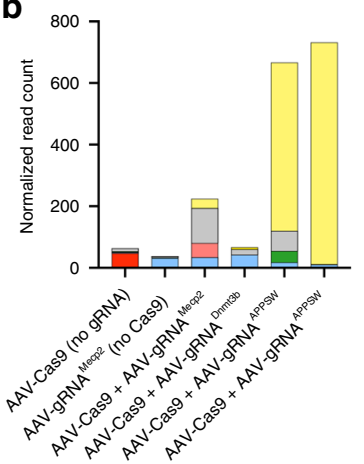

$\square$ CRISPR target

$\square$ Intergenic

Promoter/downstream/UTR

$\square$ ncRNA

$\square$ Intron

$\square$ Exon
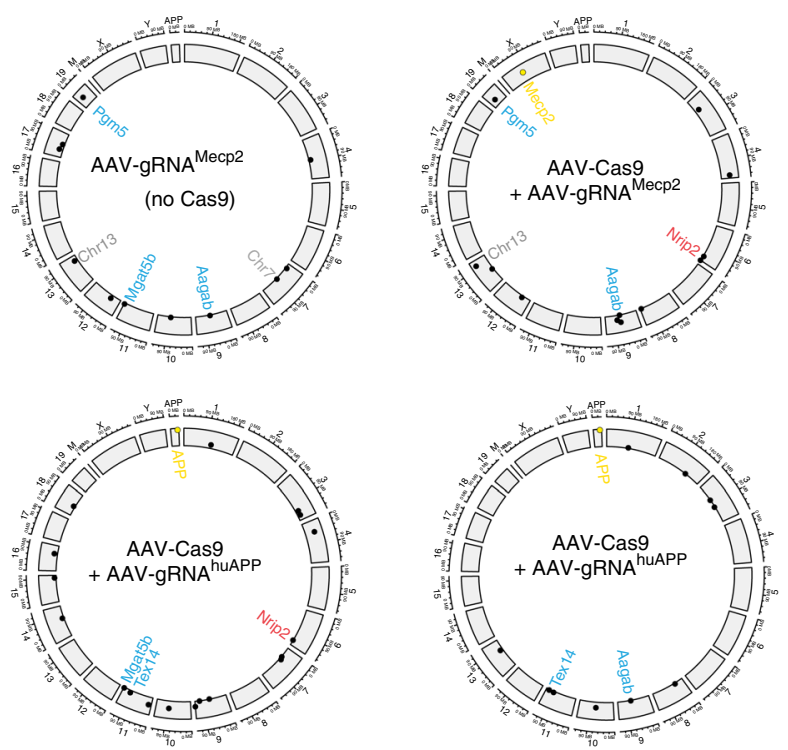

AAV-gRNA ${ }^{\text {Mecp2 }}$ (no Cas9)
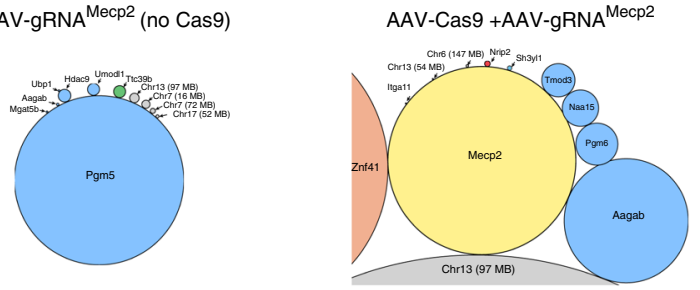

AAV-Cas9 + AAV-gRNA ${ }^{\text {APPSW }}$

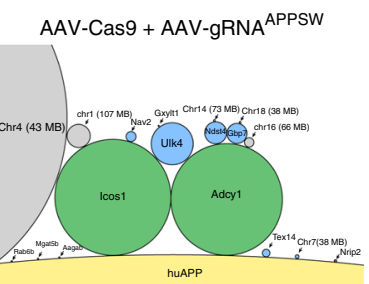

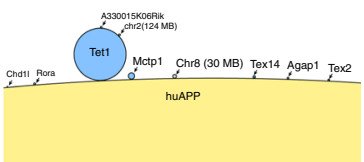

Fig. 2 Genome-wide AAV mapping from CRISPR treated mouse brains. a Total number of unique integration sites. In the case of AAV-Cas9, AAVgRNAMecp2, AAV-Cas9 + gRNAMecp2, and AAV-Cas9 + gRNA ${ }^{\text {Dnmt3b }}$, three mouse brains were pooled together for library construction. For AAV-Cas9 + gRNAAPPSW, hippocampus tissues from two animals were separately processed for library construction. The colors represent different genomic integration types and are based on the output of Virus-Clip. $\mathbf{b}$ Total number of reads that contain integrants normalized to total reads, based on the output from VirusClip. c Circos plots on showing the chromosomal location of AAV integration events. The more eccentric a dot is, the higher the normalized read count for that site is, on a logarithmic scale. The gene names inside the circle represent either CRISPR targets or sites that are common integration events (present at least in three different samples). Colors of gene names are the same as in $\mathbf{b}$. The human APP gene was added as a separate chromosome. $\mathbf{d}$ Bubble-plots showing all integration sites. The size of the circle is proportional to the normalized read count. The color was kept consistent in the figure in respect to type of integration. Intergenic integrations are marked by the chromosome and location. Source data are provided as a Source Data file 
a

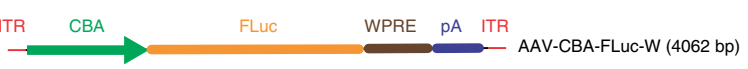

ITR $\underline{\lambda \text { ITR }}$ AAV- $\lambda 465(465 \mathrm{bp}) \quad \underline{500 \mathrm{bp}}$

b

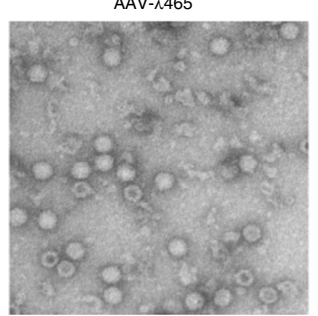

AAV-CBA-FLUC-W

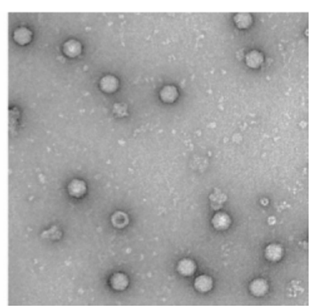

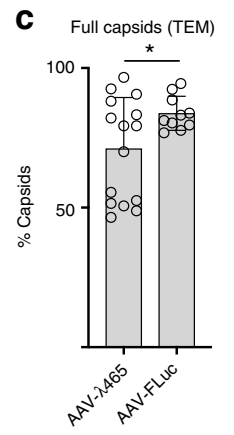

e

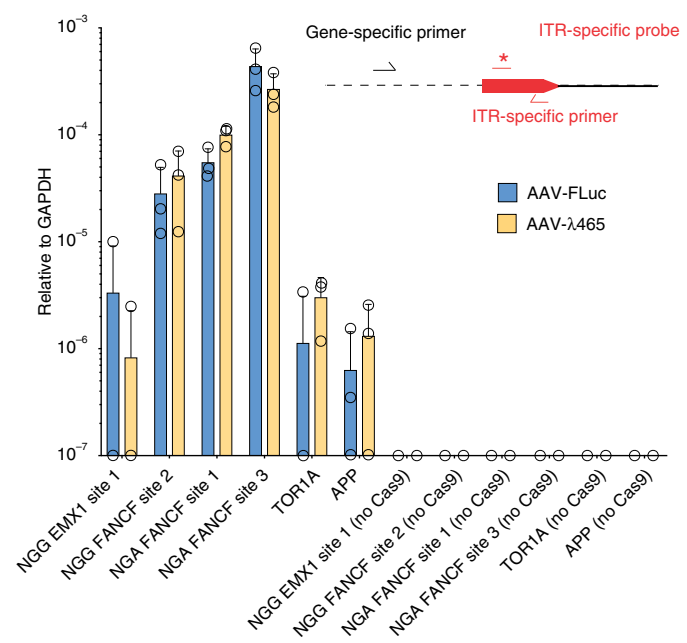

g

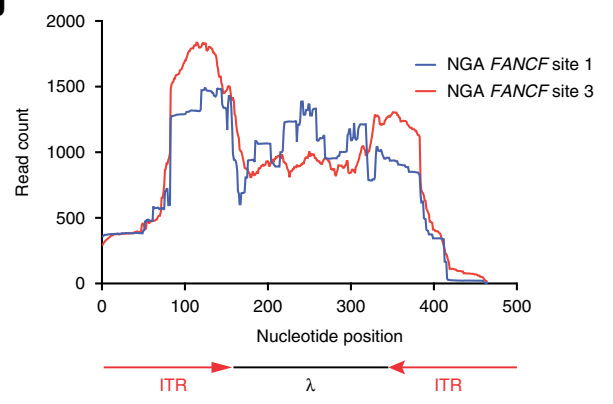

d

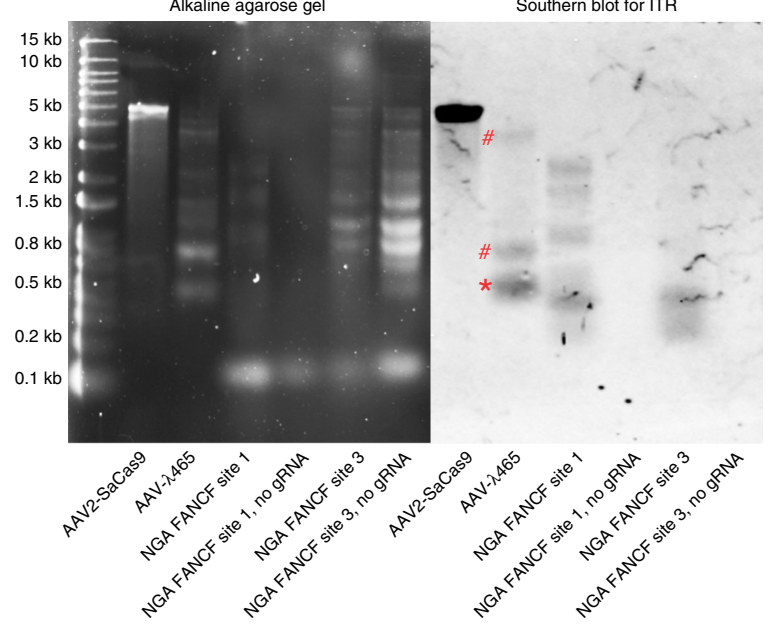

f

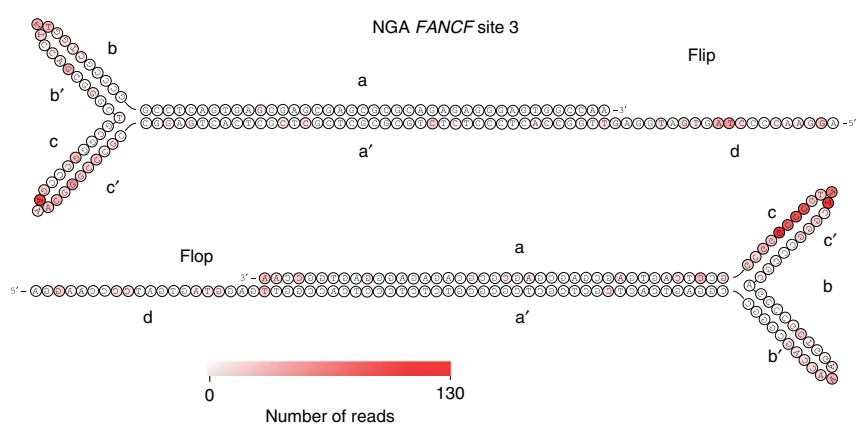

h

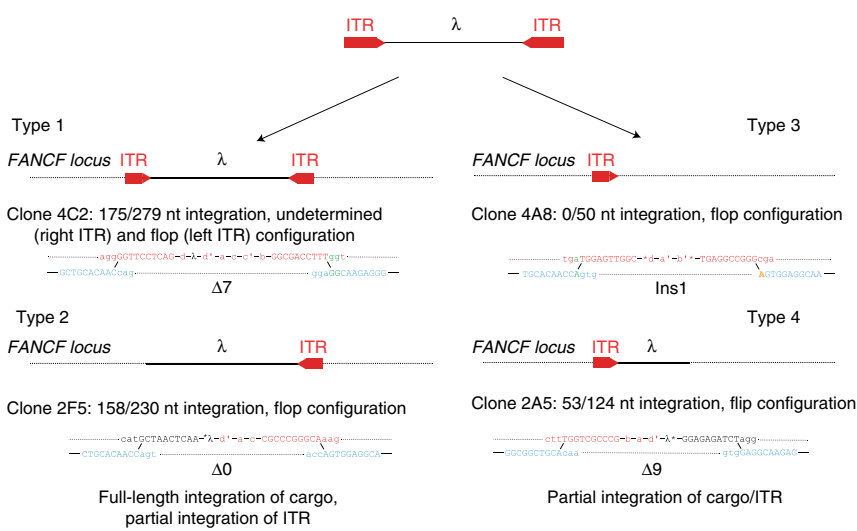

Fig. 3 Characterization of AAV vector integration into CRISPR cut sites using a miniaturized AAV genome. a Schematic of standard-sized AAV-CBA-FLuc vector (top, 4062 bases) vs miniaturized AAV- $\lambda 465$ (bottom, 465 bases). Chart is to scale. $\mathbf{b}$ Transmission electron microscopic examination of iodixanol gradient-purified capsids of AAV2- $\lambda$ or AAV2-CBA-FLuc. c quantitation of full vs empty capsids (bars represent mean \pm SEM, data from two independent experiments, 15 and 10 images were taken and 954 and 1231 capsids were counted for AAV- $\lambda 465$ and AAV-CBA-FLuc, respectively, and $p=0.0254$, unpaired $t$-test). d Alkaline gel electrophoreses and Southern blot for AAV genomes from iodixanol purified vectors (AAV2-CMV::NLS-SaCas9-NLS-3xHAbGHpA;U6::Bsal-sgRNA ( $p$ 601, $4.8 \mathrm{~kb}$ size) and AAV- $\lambda 465$ (465 bp size) and cellular genomic DNA containing integrated AAV- $\lambda 465$. For Southern blot, we used a probe specific for the ITR region. Star $\left(^{*}\right)$ highlights the $465 \mathrm{bp}$ expected band and pound (\#) sign highlights concatemers in the AAV- $\lambda 465$ genome. e ITR-genomic fusion events quantified by integration-specific qPCR assay, using AAV- $\lambda 465$ or AAV2-CBA-FLuc vectors determined (bars represent mean $\pm S D$ ). Three independent experiments were performed using two technical replicates each. $\mathbf{f}$ Heatmap of AAV specific ITR nucleotide integration at CRISPR cut site. More saturated red indicates higher frequency of breaks at the given position. $\mathbf{g}$ Integration profile of the entire miniaturized AAV genome from U2-OS cells. $\mathbf{h}$ Representative individual AAV integration clones showing different forms of integration detected (for all the clones, see Supplementary Fig. 4). Source data are provided as a Source Data file 
First, we asked whether such a short AAV transgene cassette could be packaged into AAV2 capsids during production in 293T cells. Cells were transfected with plasmids required for AAV2- $\lambda 465$ generation. As a control, we used a $4 \mathrm{~kb}$ long AAV vector encoding firefly luciferase (FLuc) driven by the chicken beta actin promoter (CBA), AAV-CBA-FLuc. First, we performed qPCR to quantitate the amount of each vector in the cell culture media using the ITR regions for probing. Titers of DNaseresistant AAV particles of AAV2- $\lambda 465$ and AAV2-CBA-FLuc were not significantly different, suggesting successful packaging of the small genome into AAV2 capsids (AAV2- $\lambda 465: 7 \times 10^{12} \pm$ $3.3 \times 10^{12} \mathrm{vg} / \mathrm{mL}\left(\right.$ mean \pm SD) and AAV2-CBA-FLuc: $7.4 \times 10^{12}$ $\pm 6.6 \times 10^{12}$ (mean $\left.\pm \mathrm{SD}\right)$, difference not significant by $t$-test). Next, we isolated and purified AAV- $\lambda$ vectors on iodixanol gradients, and achieved DNase-resistant AAV particle titers of $>$ $10^{12} \mathrm{vg} / \mathrm{ml}$. We performed transmission electron microscopy (TEM) on AAV2- $\lambda 465$ and AAV2-CBA-FLuc (Fig. 3b). AAV capsids were observed both in AAV2- $\lambda 465$ and AAV2-CBA-FLuc samples. We also counted full vs. empty capsids on TEM images and observed that AAV2- $\lambda 465$ has significantly higher empty capsids (28.8\%) compared with AAV2-CBA-FLuc (16.1\%); unpaired $t$-test, $p=0.0254$, Fig. $3 \mathrm{c}$ ). The DNase-resistant vector titers as well as TEM analysis of capsids suggests successful packaging of AAV2- $\lambda 465$. Due to the small size of the $\lambda$ cassette, we wondered if individual AAV2 capsids would package multiple miniaturized monomeric genomes. If there would be on average multiple genomes per capsid, one would expect lower number of capsids for a given amount of AAV genomes. To determine capsid/genomic copy ratio, we performed ELISA for AAV2 capsids and qPCR for AAV genomes. Capsid/genomic copy ratio was not significantly different for AAV2- $\lambda 465$ and AAV2-CBAFLuc at $10^{9} \mathrm{vg} /$ well, and was significantly increased (unpaired $t$ test, $p=0.026)$ in the case of AAV2- $\lambda 465$ compared with AAV2CBA-FLuc at $10^{10} \mathrm{vg} /$ well (Supplementary Fig. 2). These results suggest that AAV2- $\lambda 465$ on average does not harbor more than one monomeric genomic copy per capsid as compared with a fulllength AAV. However, concatemeric AAV- $\lambda 465$ genomes could potentially be packaged into a given capsid, which may not be detected by the ITR-specific qPCR used to titer the vector preparation, due to ITR recombination during concatamerization. To ascertain this possibility, we ran purified AAV2- $\lambda 465$ or purified AAV2-Cas9 (full-length control vector) on an alkaline agarose gel and stained the gel to observe the size of the genomes. As expected the full-length AAV2-Cas9 vector ran at $\sim 5 \mathrm{~kb}$. For AAV2- $\lambda 465$, a band migrating just below $0.5 \mathrm{~kb}$ indicating monomeric genomes was observed. Interestingly, additional bands between $\sim 0.7$ and $4 \mathrm{~kb}$ were observed indicating some packaged genomes were concatemers (Fig. 3d). A Southern blot using an ITR-specific probe confirmed the presence of monomeric and concatemeric genomes in the packaged AAV capsid (Fig. 3d). Importantly, we did not observe smaller bands in the case of AAV2- $\lambda 465$, suggesting no significant fragmentation of the small AAV genome.

To precisely quantify and characterize AAV integration into nuclease-induced breaks in the U2-OS human cell line using AAV2- $\lambda 465$ (or AAV2-FLuc for comparison), we supplied Cas9 and gRNA using transfected plasmids into cells after overnight transduction with the AAV vector. First, we analyzed indel formation and AAV integration in four target sites (Supplementary Table 1). All target sites that showed indel formation showed AAV capture as well, as assayed by NGS (Supplementary Table 1). We observed AAV capture rates between 3 and $38 \%$ in the case of AAV2- $\lambda 465$ (Supplementary Table 1). Next, we developed an integration-specific qPCR assay with one primer being in the ITR and one primer being in the target gene (Fig. 3e). We selected the ITR region as the priming site as the ITR was present in the junction in the vast majority on fusion reads (Fig. 1f). We assessed the presence of ITR-genomic fusion events in the case of six different genes (Fig. 3e). There was no amplification from cells treated with Cas9 plasmid and AAV vector only (i.e., no gRNA) (Fig. 3e) indicating that a site-specific DSB is required for AAV integration. In contrast, the integration-specific qPCR assay showed integration in all target genes when cells were transfected with Cas9 and gRNA plasmids, and co-transduced by AAVs. There was no difference between the GAPDH-normalized ITRgenomic fusion events between the $465 \mathrm{bp}$ AAV2- $\lambda 465$ and the $4.1 \mathrm{~kb}$ AAV2-FLuc construct in any of the target genes analyzed (Fig. 3e).

Since we observed concatemeric genomes packaged in AAV2$\lambda 465$, we analyzed integration of concatemers in U2-OS cells using alkaline gel electrophoresis and Southern blotting. Using a probe specific for the ITR region, analysis of genomic DNA from U2-OS cells treated with Cas9/gRNA and AAV2- $\lambda 465$ revealed integration into NGA FANCF site 1 and NGA FANCF site 3 (NGA is the PAM site of the SpCas9-VRQR PAM variant ${ }^{1}$ ), but there was no integration in the no gRNA control (Fig. $3 \mathrm{~d}$ ). The major integrant size was smaller than the AAV2- $\lambda 465$ genome, but we observed larger bands (up to $3 \mathrm{~kb}$ ) particularly in the case of NGA FANCF site 1, indicating concatemeric integration of AAV- $\lambda 465$.

Next, to analyze if there is a preferred breakpoint in the ITR region we determined, for each nucleotide in the ITR region, how frequently that nucleotide was represented in fusion reads at the breakpoint (i.e., breakpoint would be adjacent to this nucleotide). A heatmap (Fig. 3f) revealed that there are preferential breakpoints in the " $b$ " and " $c$ " loop regions of ITR elements. Next, we analyzed AAV2- $\lambda 465$ integration into the FANCF NGA sites 1 and $3^{24}$ using a specific gRNA, to assess whether there were any regions of the ITR which are present more often at the indels. Interestingly, in both the "flip" and "flop" ITR conformations, all ITR regions were present at relatively similar read counts (Supplementary Fig. 3). We observed a high frequency representation of the ITR elements when plotting the coverage of AAV2- $\lambda 465$ (Fig. 3g), although we also readily detected integration of the $\lambda$ sequence. Finally, in order to precisely characterize integrants and indels at the same time, we cloned amplified PCR fragments into a vector and performed Sanger sequencing of 285 individual bacterial clones from both sides of the integrant. We were able to recover 20 clones with AAV vector sequences. Integration of the $\lambda$ sequence was either full length or partial (Fig. 3h). We observed four major integration types at the NGA FANCF site 3 locus: (Type 1;2 clones recovered) both ITRs are present and the full $\lambda$ payload is detectable; (Type 2;4 clones recovered) full AAV- $\lambda$ cargo with one ITR and no second ITR; (Type 3; 5 clones recovered) ITR-only integrations; and (Type 4;9 clones recovered) one ITR with partial $\lambda$ sequences (Fig. $3 \mathrm{~h}$ and Supplementary Fig. 4). We did not detect any integrants lacking ITR sequences. The presence of microhomology regions (depicted as green nucleotides on Fig. 3h and Supplementary Fig. 4) were sometimes present, however, for several clones, no microhomology regions were observed. These data suggest that the ITR is required for integration, but only one ITR is needed for a successful capture. Furthermore, our data suggest that the majority of integration events do not contain the full-length AAV genome, however, full-length AAV integration events are also readily detected.

\section{Discussion}

Genome editing as a therapeutic tool is developing at a rapid pace, with the first in vivo clinical trials being planned ${ }^{11}$ or underway (clinicaltrials.gov: NCT03041324, NCT02702115). The 
discovery of genome editing systems allows specific targeting of genomic sequences, although gene editing outcomes are generally less predictable and are probably cell-type specific. A DSB induced by CRISPR elements will be followed by cellular DNA repair, including NHEJ, microhomology-mediated end joining (MMEJ) or homology directed repair (HDR) ${ }^{26,27}$. The presence of various DNA repair machineries and the error-prone nature of NHEJ leads to genomic heterogeneity after gene editing. It is crucial to better understand and predict gene editing outcomes in preclinical studies in order to design as safe clinical trials as possible.

In this study we set out to analyze one potential outcome of gene editing: AAV vector integration. AAV is a virus that is generally maintained in an extrachromosomal form. Wild-type $\mathrm{AAV}$ is known to integrate into the AAVS1 site of the human genome, however, this is mediated by the Rep protein and AAV vectors used in gene therapy lack the Rep protein ${ }^{28,29}$. However, even AAV vectors can integrate at a low frequency $(\sim 0.1 \%$ of total vector genomes), and studies conducted in one strain of mice found AAV insertion into the Rian locus associated with hepatocellular carcinoma formation ${ }^{30}$. This locus, however, is absent in non-human primates (NHPs) and humans, and hepatocellular carcinoma induction has not been associated with AAV vectors in NHPs and humans ${ }^{31}$. There is accumulating evidence of AAV vector genome integration into nuclease-induced breaks after radiation $^{32}$, zinc-finger nucleases ${ }^{14}$ or CRISPR/Cas9 nucleases 9,15 In this study we also show AAV integration into Cas9-induced breaks in vitro and in vivo in therapeutically relevant genes. When using a long extension time to allow for PCR amplification of large integrants, AAV capture efficiencies were found to be up to $47 \%$. We observed similar integration profiles and AAV capture rates in brain, cochlea and muscle. This suggests that AAV integration is a major gene editing outcome and has to be considered in gene editing applications. Previous studies have suggested the role of ITRs in this process of integration ${ }^{12}$ and our study confirmed this finding. Using NGS, we were able to identify the nucleotides in the ITR loop regions that are most likely to serve as breakpoints.

We next focused on AAV-Cas9/AAV-gRNA targeting therapeutically relevant genes in the brain and asked whether CRISPR can enhance genome-wide AAV integration. AAV sequencing revealed integration of the AAV cassette into all target genes analyzed (Dnmt3b, Mecp2, and $A P P S W$ ), as expected. We did not observe a significant increase in overall AAV integration when using Cas9 compared with no Cas9 present (AAV-gRNA only). This suggests that AAV-mediated expression of Cas9 does not mediate a significant genome-wide genotoxicity. This is in line with recent data showing that appropriately designed gRNAs do not mediate off-targets in vivo ${ }^{33}$. Interestingly we found short sequences in some target genes that have homology to the ITR region. The integration sites detected in the brain were different from what it has been reported in the mouse liver ${ }^{34}$ suggesting that integration is cell- or tissue specific.

Previous studies could not demonstrate that AAV integration involves the full length or only partial integration of the vector, as amplifying and sequencing through a $\sim 3-5 \mathrm{~kb}$ AAV cargo is difficult. To overcome this hurdle, we synthesized a very short AAV (465 bp), AAV- $\lambda 465$, which could be sequenced entirely after integration. This miniature AAV cassette packaged efficiently in AAV2 capsids, as assessed by qPCR titering of DNaseresistant vector particles, with an only slightly elevated fraction of empty capsids. Southern blotting of purified vector, however, revealed both monomeric $465 \mathrm{bp}$ vector as well as concatemers. We observed a band at $\sim 700 \mathrm{bp}$ probably corresponding to doublets with processed (shorter) ITR elements, but also larger concatemers up to $4 \mathrm{~kb}$.
Next, we analyzed integration of AAV- $\lambda 465$ with qPCR, NGS, Southern blotting and TA cloning. An integration-specific qPCR analysis confirmed that a CRISPR-mediated double-stranded break is required for integration. We observed no difference in ITR-genomic fusion events between AAV- $\lambda 465$ and a full-length AAV using qPCR suggesting similar integration efficiency. One could speculate that these data indicate that AAV size is not a major determinant of integration efficiency but is dependent on the presence of ITR. However, a comparison between AAV- $\lambda 465$ and a full-length AAV regarding integration efficiency is difficult due to the presence of heterogenous genomes in the minimal AAV vector. Southern blotting of genomic DNA of U2-OS cells revealed integration events of various sizes after AAV- $\lambda 465$ transduction, including shorter and longer integrants than the size of the AAV- $\lambda 465$ plus the genomic region. The shorter integrants likely correspond to fragmented AAV genomes also observed by TA cloning. The larger integrants likely represent concatemers formed either by integration of the concatemeric genomes or concatemerization of vector genomes after transduction. Despite the heterogeneous nature of AAV- $\lambda 465$, however, it did effectively characterize the integration pattern of monomeric AAV genomes, which prior studies had not achieved due to the challenges of sequencing full-sized AAV genomes.

Although standard-sized AAV vectors would not form large concatemers within a capsid, concatemerization of AAV genomes does occur during transduction. This has been well characterized in prior studies as episomal forms of $\mathrm{AAV}^{35-37}$. To our knowledge, no groups have analyzed integration of concatemers of AAV into the genome using NGS. While our genome-wide analysis did not assay for this form of integrant, it will be interesting to assess in future studies of AAV vector integration into the genome.

TA cloning of integrated AAV fragments showed various types of integration of the AAV- $\lambda 465$ cassette. We observed fulllength integration of the cargo, fragmented integrations and also ITR-only integrants. These fragments are likely not coming from input fragmented AAV genomes, as Southern blotting of purified vector did not reveal any fragmented genomes for AAV- $\lambda$ 465. Concatemers were not detected with TA cloning, however, this is probably due to the fact that PCR and TA cloning are biased toward smaller fragments. Most importantly, TA cloning did not detect any integration of the cargo sequence without the presence of at least one ITR. TA cloning also revealed indel formation in the genomic DNA indicating the activity of NHEJ machinery. The requirement of short homology regions between ITRs and genomic target regions was not apparent, with several clones lacking these short homology regions. This suggests that integration can happen independent of the repair machinery. The TA cloning results suggest a hypothesis of integration: (1) after double-stranded break, one broken ITR is captured at specific bases, (2) the other end of the AAV vector is chewed back, and (3) the remaining AAV vector fragment is ligated to the other end of the genomic DNA. In some cases, the two ITRs get captured on either end of the break leading to full-length AAV integration. The loss of nucleotides from the genomic DNA is commonly seen suggesting NHEJ mediated repair. The integration of AAV vectors at DSBs induced by CRISPR is likely to occur via similar mechanisms to that described by the seminal work of David Russell's group which showed AAV ITR mediated integration into DNAdamage induced $\mathrm{DSBs}^{32}$. Our work, similar to this prior study, observed ITR sequences present at DSBs with some clones showing microhomology between ITR and genomic DNA and others with no apparent homology. Similarly, both studies demonstrated apparent hot spots within the loop regions of the ITRs which were found at breakpoints. 
During the course of our work, a very recent publication by Nelson et al. ${ }^{15}$ described long-term efficacy of CRISPR editing for muscular dystrophy in a mouse model, and the authors also reported AAV sequences integrated at the CRISPR cut site. Our data confirm AAV integration into CRISPR-induced doublestranded breaks in other organs, including brain and cochlea. There are two major features that differentiate our work and that of Nelson et al. ${ }^{15}$. First, our use of the miniaturized AAV vector allowed us to characterize the complete profile of the AAV integration events, which had not been possible before with standard AAV where the genome size and the limits of nextgeneration sequencing prevented this from being achieved. Second, our study provides a genome-wide analysis of AAV integration in the context of CRISPR in the brain.

Taken together, we have shown that AAV integration at the on-target cut site is a major gene editing outcome in vivo and it has to be considered when assessing safety. Importantly, however, we did not detect increased genome-wide integration of AAV in the presence of CRISPR/Cas9 in the brain suggesting high target specificity. Furthermore, AAV integration could be used as a sensitive assay to detect the presence of double-stranded breaks, and, in the future, it might be applied to directly assaying genome-wide specificities of genome editing nucleases.

\section{Methods}

Plasmids and cloning. pX551 was a gift from Feng Zhang (Addgene plasmid \# 60957). pX552 was a gift from Feng Zhang (Addgene plasmid \# 60958). pX601 was a gift from Feng Zhang (Addgene plasmid \# 61591). Supplementary Table 2 has information on the plasmids used in this study. Cloning of gRNAs (Supplementary Table 3) into pX552 was performed with FastDigest LguI (Thermo Scientific) and verified by Sanger sequencing using a U6 sequencing primer: 5'-GACTATCATATGCTTACCGT- $3^{\prime}$. To create AAV2- $\lambda 465$, GenScript (Piscataway, NJ) synthesized the $\lambda$ fragment using sequence from Enterobacteria phage lambda, (NC_001416.1) flanked by KpnI and SphI sites (for the sequence see Supplementary Data 17). We cloned two different length fragments into a single stranded AAV-CBA-W empty backbone cut with KpnI and SphI. The final vector has only a short linker, the sequence of two GUIDE-Seq oligos and a $70 \mathrm{nt}$ fragment from the $\lambda$ phage (Supplementary Data 17). The total length of the AAV vector including the two ITRs on either end were $465 \mathrm{bp}$. Cloning was validated by running KpnI/SphI digested vector on a gel and Sanger sequencing.

AAV production. For production of AAV vectors, we used the triple transfection method. Briefly, we plated ten $15-\mathrm{cm}$ tissue culture dishes with $1.5 \times 10^{7}$ HEK293 T cells (ATCC ${ }^{\circledR}$ CRL-3216) per dish. The next day, cells were transfected using the calcium phosphate method, with the adenovirus helper plasmid (pAd $\Delta \mathrm{F} 6,26$ $\mu \mathrm{g}), \mathrm{rep} / \mathrm{cap}$ plasmid (e.g., AAV2 or AAV1, $12 \mu \mathrm{g}$ ), and ITR-flanked transgene cassette plasmid $(10 \mu \mathrm{g})$, per plate, to induce production of AAV (pX551, pX552, AAV- $\lambda 465$, AAV-CBA-FLuc-WPRE). The day after transfection, medium was changed to DMEM containing $2 \%$ fetal bovine serum (FBS). AAV was purified from the cell lysate using iodixanol density-gradient ultracentrifugation. Buffer exchange to PBS was conducted using Zeba spin desalting columns (7 K MWCO; Thermo Fisher Scientific) and further concentration was performed using Amicon Ultra 100-kDa MWCO ultrafiltration centrifugal devices (Millipore). Vectors were stored at $80^{\circ} \mathrm{C}$ until use. We quantified AAV vector genomes in AAV preparations using TaqMan qPCR with primer and probes specific to the ITR region of the virus.

Transmission electron microscopy. Five microliters of the AAV sample was adsorbed for $1 \mathrm{~min}$ to a carbon-coated grid (EMS) that had been made hydrophilic by a $20 \mathrm{~s}$ exposure to a glow discharge $(25 \mathrm{~mA})$. AAVs were stained with $1 \%$ uranyl acetate (EMS catalog \# 22400) for 20-30 s. After removing the excess stain with a filter paper, the grids were examined in a TecnaiG ${ }^{2}$ Spirit BioTWIN and images were recorded with an AMT 2k CCD camera at the Harvard Medical School Electron Microscopy Facility.

Capsid ELISA. ELISA plates were coated with A20 anti-AAV2 antibody $(0.1 \mu \mathrm{g} /$ well $)$ in bicarbonate buffer overnight. Plates were washed twice with PBS, then blocked blocking solution (Thermo, Casein blocking solution in TBS: Blocker Casein in TBS, $100 \mathrm{~mL}, 1 \%$ (w/v) casein (Hammarsten grade) in $25 \mathrm{mM}$ Tris, $150 \mathrm{mM} \mathrm{NaCl}$, $\mathrm{pH} 7.4$ containing Kathon Anti-microbial Agent, cat no: 37583 ) for $1 \mathrm{~h}$ at $37^{\circ} \mathrm{C}$. In total, $1 \times 10^{9}$ and $1 \times 10^{10}$ vector genomes of AAV- $\lambda 465$ and AAV-CBA-FLuc were added to the plate in $100 \mu \mathrm{L}$ volume and plates were incubated for $90 \mathrm{~min}$ at $37^{\circ} \mathrm{C}$. After washing three times with PBS-Tween, samples were incubated in the presence of a 1:20 dilution of primary antibody (Anti-AAV2, intact particles, clone
A20, BIOTIN, American Research Products, Waltham, MA; Product Code: 03 $61055 \mathrm{~B})$ in $50 \mu \mathrm{L}$ volume. After washing three times with PBS-Tween, 1:2000 streptavidin-HRP was added (GE Healthcare, RPN1231V) and plates were incubated for $1 \mathrm{~h}$ at $37^{\circ} \mathrm{C}$. After the addition of TMB substrate, and $\mathrm{H}_{2} \mathrm{SO}_{4}$, absorbance was read at $450 \mathrm{~nm}$. Samples were run in duplicates.

Cell culture and nucleofection. U2-OS cells (Homo sapiens bone osteosarcoma, ATCC ${ }^{\otimes}$ HTB-96 ${ }^{\mathrm{m}}$ ) were cultured in Dulbecco's modified Eagles media (DMEM) supplemented with $10 \%$ bovine serum albumin und $1 \%$ penicillin/streptomycin. We performed Mycoplasma testing on a monthly basis using the MycoAlert Kit (Lonza). Cells were transfected with the Nucleofector device (Lonza) using CM-104 program and the SE kit. For each transfection, we used 500 ng Cas 9 plasmid and $250 \mathrm{ng}$ gRNA plasmid (see Supplementary Table 2 for plasmid specification for each gene)

TA cloning. U2-OS cells were treated with AAV- $\lambda 465$ and were transfected with SpCas9-VRQR variant and gRNA against NGA FANCF site 3 (see details of transfection above). Genomic DNA was isolated by the Qiagen Blood and Tissue Kit, and 100 ng DNA was subjected to targeted PCR (see primers in Supplementary Table 4) using the Phusion polymerase (NEB). The samples were subjected to gel purification (gel was cut above the expected $132 \mathrm{bp}$ PCR band and below $\sim 600 \mathrm{bp}$ ). After gel purification, DNA sample was ethanol precipitated for $20 \mathrm{~min}$ at $-20^{\circ} \mathrm{C}$ and $1 \mu \mathrm{L}$ reaction $(12 \mathrm{ng}$ ) was used to in the TA cloning reaction according to manufacturer's instructions $\left(\mathrm{TOPO}^{\circledR}\right.$ TA Cloning Kit for Sequencing, ThermoFisher). Three full plates (285 clones) were sequenced with the T7 primer included in the cloning kit.

Neuronal culture. In this study, we performed original experiments and for some cases we reanalyzed existing sequencing data from our previous publications. A detailed description of every data point is found in the Source Data file. For this study, wild-type C57BL/6 or C57BL/6:SJL male mice (see Source Data file for details) were housed with wild-type females for 3 days to generate timed pregnancies for embryo-derived, primary neuronal cultures ${ }^{8}$. At embryonic days $14-17$, the females were euthanized by $\mathrm{CO}_{2}$ and the embryos were collected. After dissection, the embryonic cortices were collected separately in Hank's Balanced Salt Solution with $1 \%$ penicillin (Pe)/streptomycin (St) and $10 \mathrm{mM}$ HEPES buffer, $\mathrm{pH}$ 7.4 (HBSS) and placed on ice. The cortices were centrifuged at $200 \times g$ for $5 \mathrm{~min}$ and dissociated in $500 \mu \mathrm{L}$ Plating media (Neurobasal media-with $1 \% \mathrm{Pe} / \mathrm{St}, 10$ mM HEPES buffer, 1xGlutaMAX, and 10\% fetal bovine serum (FBS). Approximately 300,000 cells were plated in each well of 12 well, tissue treated, plastic plates (Corning, Inc.) coated with Poly-L-ornithine (1:4 in $\mathrm{H}_{2} \mathrm{O}$, Sigma) and laminin (1:1000 in PBS). After $5 \mathrm{~h}$, the media was replaced with Neurobasal media, similar to the plating media but containing serum-free B-27 supplement instead of FBS. At day 3, the neurons were treated with AAV1-pMecp2-SpCas9-spA (referred to as AAV-Cas9) mixed with AAV1-U6sgRNA(SapI) hSyn-GFP-KASH-bGH (referred to as AAV-gRNA). We added $10^{5}$ genomic copies per cell with a 1:2 AAV-Cas9 to AAV-gRNA ratio. Control cells were transduced with AAV-Cas9 alone without gRNA. DNA was isolated as described at 21 days in vitro (DIV). All products for neuronal culture were purchased from Thermo Fisher Scientific if not otherwise stated.

Animals. In this study, we performed original experiments and for some cases we reanalyzed existing sequencing data from our previous publications. A detailed description of every data point is found in the Source Data file. For this study, 8-10-week-old male C57BL/6 mice were used in this study. Animals were purchased from Charles River Laboratories. We attest that we have complied with all relevant ethical regulations for animal testing and research. The research described in this study was approved by Animal Care Committee and Partners Institutional Biosafety Committee of Partners Healthcare (Massachusetts General Hospital, Boston, MA, USA).

In vivo injections and DNA isolation. Mice were anesthetized by intraperitoneal (i.p.) injection of $100 \mathrm{mg} / \mathrm{kg}$ ketamine and $10 \mathrm{mg} / \mathrm{kg}$ xylazine. A small burr hole was drilled in the skull and mice were stereotactically injected with a mixture of AAV1-Mecp2-Cas9 $\left(5 \times 10^{9} \mathrm{vg}\right)$ and AAV1-U6-gRNA ${ }^{\text {Mecp2 }}$-hSyn-GFP or AAV1-

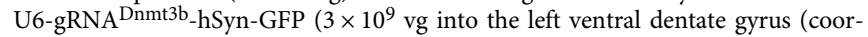
dinates: anterior/posterior $(y):-3.5$, mediolateral $(x):+2.7$, dorsal/ventral $(z):-3)$ We injected using a Hamilton syringe fitted with a 30 gauge needle at a rate of 0.2 $\mu \mathrm{l} / \mathrm{min}$ using a pump and controller (World Precision Instruments, Sarasota, FL) Six weeks after injection, genomic DNA was isolated directly from hippocampus using the Qiagen Blood and Tissue Kit (Qiagen). Briefly, we added $360 \mu \mathrm{L}$ of ATL buffer and $40 \mu \mathrm{L}$ of proteinase $\mathrm{K}$ to dissected hippocampus tissue. Samples were then digested for $5 \mathrm{~min}$ at $56^{\circ} \mathrm{C}$ and were homogenized using disposable pestles. Next, samples were digested for another $10 \mathrm{~min}$ at $56^{\circ} \mathrm{C}$. After vortexing, we added $400 \mu \mathrm{L}$ of the AL buffer from the kit and incubated the samples for another $5 \mathrm{~min}$ at $56{ }^{\circ} \mathrm{C}$. After the addition of $400 \mu \mathrm{L}$ of $100 \%$ ethanol, we purified the genomic DNA according to the manufacturers' instructions. 
Targeted DNA Sequencing. Genomic DNA from U2-OS cells, cultured cortical neurons, or brain tissue was PCR amplified using Phusion polymerase (NEB) according to the manufacturer's instructions. Importantly, we kept the extension time long $(4: 15 \mathrm{~min})$ so that even several kilobases could be amplified. PCR products were visualized on a $1 \%$ agarose gel using GelRed (Thermo Fisher) and purified on a column (PCR Purification Kit, Qiagen). PCR primers are given in Supplementary Table 4 . For muscle samples, we separately amplified the targeted region in intron 51, in intron 53 and exon 53, as described previously ${ }^{6}$. We also assumed the deletion of exons 52-53 and therefore we performed a PCR reaction with a primer in intron 51 (DS-i51, forward) and intron 53 (DS-i53, reverse). For targeted deep sequencing we used 500-1400 ng genomic DNA. Sequencing was performed at the MGH DNA Core (Sanger sequencing and CRISPR-sequencing service). Paired-end reads ( $150 \mathrm{bp}$ ) were generated on an Illumina MiSeq platform with a $100 \mathrm{~K}$ read depth per sample.

Genome-wide AAV sequencing (AAV-Seq). Samples were pooled from three animals for NGS library construction in the case of Mecp2 and Dnmt $3 b$ groups. For the $A P P^{S W}$ group, we analyzed two animals separately. As controls, we included three animals injected with AAV-Mecp2-Cas9 vector only and AAV-gRNA Mecp2hSyn-GFP vector only. To amplify AAV from the genome, we used the GUIDE-Seq method adapted to AAV ITR sequences. Primer sequences and illustrated ITR priming sites are depicted in (Supplementary Fig. 1).

Targeted deep sequencing analysis. Targeted deep sequencing data was analyzed using CRISPResso (Accession code, GEO: GSE78729) ${ }^{38}$ as previously described. Briefly, reads were split into reads 1 and 2 and then merged using flash v1.2.11 with default parameters. Next, CRISPResso was run with the following parameters: CRISPResso -rl<fastq_file $>$--split_paired_end -w 5 -c $<$ protein_coding_sequence $>$ -ignore substitutions -a $<$ amplicon_sequence $>$-g $<$ gRNA sequence $>$. Substitutions were ignored. To analyze the number of AAV integrants, sequencing files were aligned to AAV vector sequences using bwa (version 0.7.17-r1188), sorted with Samtools (version 1.7) and the number of reads that had vector sequences were quantified.

\begin{abstract}
Analysis of genome-wide integration of AAV vector sequences (AAV-Seq). In order to determine the extent of genome-wide AAV vector integration, DNA from mice injected with AAV-Cas9 and AAV-gRNA was sequenced paired-end on an Illumina MiSeq, with an average read depth of 3 million reads per sample. Samples were analyzed using a viral integration detection pipeline, Virus-Clip (version 1.0) ${ }^{25}$. Briefly, Virus-Clip maps FASTQ reads from sequenced samples to indexed viral genomes of interest, using bwa (version 0.7.17-r1188). It extracts CIGAR strings from this analysis to identify sequence reads with a mix of viral and non-viral DNA. These reads are then mapped to a BLAST database of the host genome (in this case, the current mouse genomic build GRCm $38 / \mathrm{mm} 10$ ) to identify breakpoint regions containing both mouse and vector DNA, which are assigned as sites of viral integration. These sites are annotated using ANNOVAR (version 2018Apr16) ${ }^{39}$ and outputted. In this manner, sequence reads that are not fusion reads, that is, purely episomal AAV DNA, are excluded from analysis. Manual curation was then applied to exclude putative integration sites that feature any sequences common to both mouse and vector genomes. Default Virus-Clip run parameters were used. From the Virus-Clip output file, we manually analyzed all sites and excluded false hits. We observed that in all animals injected with AAV-Mecp2-Cas9, the promoter region of $M e c p 2$ was flagged as a false integration site. Similarly, the gene Syne2 was present in all samples except the AAV-Mecp2-Cas9 group. This gene showed similarity to the hSyn promoter driving GFP expression in the gRNA vector. In the next step, putative integration sites were analyzed to exclude sites that show either no ITR integration or did not appear to be bona fide fusion reads. To avoid the inclusion of genome-only regions amplified by the primers, only those sites were considered for which we were able to detect unique AAV ITR regions outside of the primer binding sites fused with genomic DNA. Fusion reads between genomic DNA and AAV were visualized using Geneious Prime 2019.0.4 (Supplementary Data 16).
\end{abstract}

Alkaline gel electrophoresis and Southern blotting. Two hundred microliters of volume containing AAV genomes $\left(3 \times 10^{12} \mathrm{vg}\right.$ for AAV2-pX601 and $2 \times 10^{11} \mathrm{vg}$ for AAV2- $\lambda 465$ ) were purified using High Pure Viral Nucleic Acid kit (Roche, Boulogne- Billancourt, France). DNase I treatment was not required because the crude cell lysates are treated with Benzonase during AAV vector purification and it is sufficient to remove plasmid DNA ${ }^{40}$. PCR products of NGA FANCF site 1 and NGA FANCF site 3 were purified on a column (Qiagen). Alkaline gel electrophoresis was performed as described previously ${ }^{41}$ with some modifications. The gel was run for $225 \mathrm{~min}$ at $30 \mathrm{~V}$ and staining was performed with SYBR Gold Nucleic Acid Gel Stain at 1:10,000 dilution. Southern blotting was performed as described originally by Ed Southern ${ }^{42}$. Briefly, we soaked the gel in alkaline transferbuffer $(0.4 \mathrm{M} \mathrm{NaOH}$ and $1 \mathrm{M} \mathrm{NaCl})$ twice for $20 \mathrm{~min}$. The blotting was performed overnight onto a positively charged membrane (Hybond-N $+(20 \mathrm{~cm} \times 3 \mathrm{~m}) \mathrm{GE}$ Life Sciences, RPN203B). Next, the membrane was neutralized using neutralization buffer (0.5 M Tris-Cl, $1 \mathrm{M} \mathrm{NaCl}, \mathrm{pH}$ 7.2) for $15 \mathrm{~min}$ and blocked for $2 \mathrm{~h}$ using Denhardt's solution (Thermo Fisher). To detect the ITR in AAV vectors and PCR products, we used a biotinylated ITR probe (5'-CACTCCCTCTCTGCGCGCTCG$3^{\prime}, 2.3 \mu \mathrm{L}$ of probe was used from a $100 \mathrm{mM}$ stock solution). Hybridization was performed in $6 \times$ saline-sodium citrate buffer (SSC, $0.9 \mathrm{M} \mathrm{NaCl}, 0.9 \mathrm{M}$ sodium citrate tribasic dehydrate). After washing twice in $2 \times$ and twice in $0.1 \times$ SSC buffer, the membrane was developed with a Chemiluminescent Nucleic Acid Detection Module Kit (Thermo) and imaged with an iBright Imaging System (Thermo).

Statistics. We used GraphPad Prism 7.0 for Mac OS and OriginPro (2015) for statistical analysis. To compare means, we used an unpaired two tailed $t$-test (after Shapiro-Wilk normality testing) or paired $t$-test; $p$ values $<0.05$ were accepted as significant.

Reporting summary. Further information on research design is available in the Nature Research Reporting Summary linked to this article.

\section{Data availability}

Raw sequencing files have been be uploaded to NCBI's Sequence Read Archive (SRA, Bioproject ID: PRJNA563918). A list of uploaded files including SRA IDs are listed in Source Data File. Detailed data analysis is available in the Supplementary tables and Supplementary Data published with this paper. The plasmid containing the AAV2- $\lambda 465$ is available upon completion of a standard Material Transfer Agreement with The Massachusetts General Hospital. A reporting summary for this Article is available as a Supplementary Information file. The source data underlying Figs. 1a, 1b, 1d, 1f, 1g, 2, 3c, 3e, 3g, Supplementary Figs. 2 and 3 and Supplementary Table 1 are provided as a Source Data file. Any other raw data that support the findings of this study are available from the corresponding author.

\section{Code availability}

All sequence analysis was run using publicly available programs, which are referred to and referenced in the paper along with run parameters where relevant. Quantification of integrant numbers was performed with a script attached to this paper as Supplementary Data 18, which also utilized publicly available software.

Received: 13 May 2019; Accepted: 11 September 2019; Published online: 30 September 2019

\section{References}

1. Kleinstiver, B. P. et al. High-fidelity CRISPR-Cas9 nucleases with no detectable genome-wide off-target effects. Nature 529, 490-495 (2016).

2. Slaymaker, I. M. et al. Rationally engineered Cas9 nucleases with improved specificity. Science 351, 84-88 (2016).

3. Chen, J. S. et al. Enhanced proofreading governs CRISPR-Cas9 targeting accuracy. Nature 550, 407-410 (2017).

4. Swiech, L. et al. In vivo interrogation of gene function in the mammalian brain using CRISPR-Cas9. Nat. Biotechnol. 33, 102-106 (2015).

5. Gao, X. et al. Treatment of autosomal dominant hearing loss by in vivo delivery of genome editing agents. Nature 553, 217-221 (2018).

6. Bengtsson, N. E. et al. Muscle-specific CRISPR/Cas9 dystrophin gene editing ameliorates pathophysiology in a mouse model for Duchenne muscular dystrophy. Nat. Commun. 8, 14454 (2017).

7. Amoasii, L. et al. Gene editing restores dystrophin expression in a canine model of Duchenne muscular dystrophy. Science https://doi.org/10.1126/ science.aau1549 (2018).

8. György, B. et al. CRISPR/Cas9 mediated disruption of the Swedish APP allele as a therapeutic approach for early-onset Alzheimer's disease. Mol. Ther.Nucleic Acids 11, 429-440 (2018).

9. McCullough, K. T. et al. Somatic gene editing of GUCY2D by AAV-CRISPR/ Cas9 alters retinal structure and function in mouse and macaque. Hum. Gene Ther. https://doi.org/10.1089/hum.2018.193 (2018).

10. Ruan, G. X. et al. CRISPR/Cas9-mediated genome editing as a therapeutic approach for leber congenital amaurosis 10. Mol. Ther. https://doi.org/ 10.1016/j.ymthe.2016.12.006 (2017).

11. Maeder, M. L. et al. Development of a gene-editing approach to restore vision loss in Leber congenital amaurosis type 10. Nat. Med. https://doi.org/10.1038/ s41591-018-0327-9 (2019).

12. Miller, D. G., Petek, L. M. \& Russell, D. W. Adeno-associated virus vectors integrate at chromosome breakage sites. Nat. Genet. 36, 767-773 (2004).

13. Miller, D. G., Petek, L. M. \& Russell, D. W. Human gene targeting by adenoassociated virus vectors is enhanced by DNA double-strand breaks. Mol. Cell. Biol. https://doi.org/10.1128/mcb.23.10.3550-3557.2003 (2003).

14. Anguela, X. M. et al. Robust ZFN-mediated genome editing in adult hemophilic mice. Blood https://doi.org/10.1182/blood-2013-04-497354 (2013). 
15. Nelson, C. E. et al. Long-term evaluation of AAV-CRISPR genome editing for Duchenne muscular dystrophy. Nat. Med. 25, 427 (2019).

16. Vreugde, S. et al. Beethoven, a mouse model for dominant, progressive hearing loss DFNA36. Nat. Genet. 30, 257-258 (2002).

17. Chen, R. Z., Akbarian, S., Tudor, M. \& Jaenisch, R. Deficiency of methyl-CpG binding protein-2 in CNS neurons results in a Rett-like phenotype in mice. Nat. Genet. https://doi.org/10.1038/85906 (2001).

18. Feng, J. et al. Dnmt1 and Dnmt3a maintain DNA methylation and regulate synaptic function in adult forebrain neurons. Nat. Neurosci. https://doi.org/ $10.1038 / \mathrm{nn} .2514$ (2010).

19. Long, C. et al. Postnatal genome editing partially restores dystrophin expression in a mouse model of muscular dystrophy. Science https://doi.org/ 10.1126/science.aad5725 (2016).

20. Tabebordbar, M. et al. In vivo gene editing in dystrophic mouse muscle and muscle stem cells. Science https://doi.org/10.1126/science.aad5177 (2016).

21. Nelson, C. E. et al. In vivo genome editing improves muscle function in a mouse model of Duchenne muscular dystrophy. Science https://doi.org/ 10.1126/science.aad5143 (2016).

22. György, B. et al. Allele-specific gene editing prevents deafness in a model of dominant progressive hearing loss. Nat. Med. 25, 1123-1130 (2019).

23. Kleinstiver, B. P. et al. Broadening the targeting range of Staphylococcus aureus CRISPR-Cas9 by modifying PAM recognition. Nat. Biotechnol. 33, 1293-1298 (2015).

24. Tsai, S. Q. et al. GUIDE-seq enables genome-wide profiling of off-target cleavage by CRISPR-Cas nucleases. Nat. Biotechnol. 33, 187-198 (2015).

25. Ho, D. W., Sze, K. M. \& Ng, I. O. Virus-Clip: a fast and memory-efficient viral integration site detection tool at single-base resolution with annotation capability. Oncotarget https://doi.org/10.18632/oncotarget.4187 (2015).

26. Ann Ran, F. et al. Genome engineering using the CRIPR-Cas9 system. Nature https://doi.org/10.1038/nprot.2013.143 (2013).

27. Kim, S. Il et al. Microhomology-assisted scarless genome editing in human iPSCs. Nat. Commun. https://doi.org/10.1038/s41467-018-03044-y (2018).

28. Samulski, R. J. et al. Targeted integration of adeno-associated virus (AAV) into human chromosome 19. EMBO J. 10, 3941-3950 (1991).

29. McCarty, D. M., Young, S. M. \& Samulski, R. J. Integration of adenoassociated virus (AAV) and recombinant AAV vectors. Annu. Rev. Genet. https://doi.org/10.1146/annurev.genet.37.110801.143717 (2004).

30. $\mathrm{Li}, \mathrm{Y}$. et al. Induction of hepatocellular carcinoma by in vivo gene targeting Proc. Natl Acad. Sci. USA https://doi.org/10.1073/pnas.1117032109 (2012).

31. Gil-Farina, I. et al. Recombinant AAV integration is not associated with hepatic genotoxicity in nonhuman primates and patients. Mol. Ther. https:// doi.org/10.1038/mt.2016.52 (2016).

32. Miller, D. G., Petek, L. M. \& Russell, D. W. Adeno-associated virus vectors integrate at chromosome breakage sites. Nat. Genet. https://doi.org/10.1038 ng1380 (2004).

33. Akcakaya, P. et al. In vivo CRISPR editing with no detectable genome-wide off-target mutations. Nature https://doi.org/10.1038/s41586-018-0500-9 (2018).

34. Chandler, R. J. et al. Vector design influences hepatic genotoxicity after adenoassociated virus gene therapy. J. Clin. Invest. https://doi.org/10.1172/JCI79213 (2015).

35. Schnepp, B. C., Clark, K. R., Klemanski, D. L., Pacak, C. A. \& Johnson, P. R. Genetic fate of recombinant adeno-associated virus vector genomes in muscle. J. Virol. https://doi.org/10.1128/jvi.77.6.3495-3504.2003 (2003).

36. Penaud-Budloo, M. et al. Adeno-associated virus vector genomes persist as episomal chromatin in primate muscle. J. Virol. https://doi.org/10.1128/ jvi.00649-08 (2008)

37. Schnepp, B. C., Jensen, R. L., Chen, C.-L., Johnson, P. R. \& Clark, K. R. Characterization of adeno-associated virus genomes isolated from human tissues. J. Virol. https://doi.org/10.1128/jvi.79.23.14793-14803.2005 (2005).

38. Pinello, L. et al. CRISPResso: sequencing analysis toolbox for CRISPR-Cas9 genome editing. Nat. Biotechnol. 34, 695-697 (2016).

39. Wang, K., Li, M. \& Hakonarson, H. ANNOVAR: functional annotation of genetic variants from high-throughput sequencing data. Nucleic Acids Res. https://doi.org/10.1093/nar/gkq603 (2010).

40. György, B. et al. Rescue of hearing by gene delivery to inner-ear hair cells using exosome-associated AAV. Mol. Ther. 25, 379-391 (2017).
41. Pryadkina, M. et al. A comparison of AAV strategies distinguishes overlapping vectors for efficient systemic delivery of the $6.2 \mathrm{~kb}$ Dysferlin coding sequence. Mol. Ther.-Methods Clin. Dev. https://doi.org/10.1038/mtm.2015.9 (2015). 42. Southern, E. Southern blotting. Nat. Protoc. 1, 518-525 (2006).

\section{Acknowledgements}

This work was supported in part by NIH NIDCD R01DC017117 (C.A.M.), NIH R01 AR44533 (N.E.B. and J.S.C.), NIH RM1 HG009490 (J.K.J.), NIH R35 GM118158 (J.K.J.) and NIH R00 CA218870 (B.P.K.). B.G. was supported by an Edward R. and Anne G. Lefler Center Postdoctoral Fellowship. M.P.Z. received a scholarship from the Kosciuszko Foundation and is thankful for the support of the Poznan University of Medical Sciences.

\section{Author contributions}

B.G. conceived of the study. B.G., K.S.H., C.A.M., X.O.B., D.P.C., B.P.K., M.P.Z., S.T. and J.K.J. designed the research study. B.G., K.S.H., S.P.G., A.V., M.P.Z., A.A.S., N.E.B., C.L., M.I., J.S.C., S.E.S., A.M. and M.A. performed experiments and analyzed data. B.G., C.A.M., X.O.B., B.P.K. and K.S.H. wrote the paper with input from all of the authors.

\section{Competing interests}

The authors declare the following competing interests. J.K.J. has financial interests in Beam Therapeutics, Editas Medicine, Excelsior Genomics, Pairwise Plants, Poseida Therapeutics, Transposagen Biopharmaceuticals, and Verve Therapeutics (f/k/a Endcadia). J.K.J. is a member of the Board of Directors of the American Society of Gene and Cell Therapy. B.P.K. and J.K.J. are inventors on various patents and patent applications that describe gene editing and epigenetic editing technologies. S.Q.T. is a co-inventor on patents and patent applications describing gene editing technologies and methods for defining their genome-wide activities. S.Q.T. is a member of the scientific advisory board of Kromatid Inc. J.S.C and N.E.B are co-inventors on muscle-specific gene editing patents; JSC has financial interests in Solid Biosciences. C.A.M. has a financial interest in Chameleon Biosciences, Inc., a company developing an enveloped adeno-associated virus (AAV) vector platform technology for repeated dosing of systemic gene therapy. M.J.A. holds equity in Excelsior Genomics. J.K.J, C.A.M., and M.J.A.'s interests were reviewed and are managed by Massachusetts General Hospital and Partners HealthCare in accordance with their conflict of interest policies. All other authors declare no competing interests.

\section{Additional information}

Supplementary information is available for this paper at https://doi.org/10.1038/s41467 019-12449-2.

Correspondence and requests for materials should be addressed to C.A.M. or B.Gör.

Peer review information Nature Communications thanks Deniz Dalkara and the other, anonymous, reviewer(s) for their contribution to the peer review of this work.

Reprints and permission information is available at http://www.nature.com/reprints

Publisher's note Springer Nature remains neutral with regard to jurisdictional claims in published maps and institutional affiliations.

\footnotetext{
Open Access This article is licensed under a Creative Commons Attribution 4.0 International License, which permits use, sharing adaptation, distribution and reproduction in any medium or format, as long as you give appropriate credit to the original author(s) and the source, provide a link to the Creative Commons license, and indicate if changes were made. The images or other third party material in this article are included in the article's Creative Commons license, unless indicated otherwise in a credit line to the material. If material is not included in the article's Creative Commons license and your intended use is not permitted by statutory regulation or exceeds the permitted use, you will need to obtain permission directly from the copyright holder. To view a copy of this license, visit http://creativecommons.org/ licenses/by/4.0/.
}

(c) The Author(s) 2019 